\title{
Magnetic Proximity Effect Features in Antiferromagnetic/Ferrimagnetic Core-Shell Nanoparticles
}

\author{
I. V. Golosovsky, ${ }^{1}$ G. Salazar-Alvarez, ${ }^{2,3}$ A. López-Ortega, ${ }^{2,4}$ M. A. González, ${ }^{5}$ J. Sort, ${ }^{6}$ M. Estrader, ${ }^{2}$ S. Suriñach, \\ M. D. Baró, ${ }^{4}$ and J. Nogués ${ }^{7}$ \\ ${ }^{1}$ St. Petersburg Nuclear Physics Institute, 188300, Gatchina, St. Petersburg, Russia \\ ${ }^{2}$ Centre d'Investigació en Nanociència i Nanotecnologia (ICN-CSIC), Campus Universitat Autònoma de Barcelona, \\ E-08193 Bellaterra, Spain \\ ${ }^{3}$ Materials Chemistry Group, Department of Physical, Inorganic, and Structural Chemistry, Arrhenius Laboratory, \\ Stockholm University, S-106 91 Stockholm, Sweden \\ ${ }^{4}$ Departament de Física, Universitat Autònoma de Barcelona, E-08193 Bellaterra, Spain \\ ${ }^{5}$ Institut Laue Langevin, 6 rue Jules Horowitz, BP 156, F-38042 Grenoble, France \\ ${ }^{6}$ Institució Catalana de Recerca i Estudis Avançats (ICREA) and Departament de Física, Universitat Autònoma de Barcelona, \\ E-08193 Bellaterra, Spain \\ ${ }^{7}$ Institució Catalana de Recerca i Estudis Avançats (ICREA) and Centre d'Investigació en Nanociència i Nanotecnologia (ICN-CSIC), \\ Campus Universitat Autònoma de Barcelona, E-08193 Bellaterra, Spain
}

(Received 8 December 2008; published 16 June 2009)

A study of "inverted" core-shell, $\mathrm{MnO} / \gamma-\mathrm{Mn}_{2} \mathrm{O}_{3}$, nanoparticles is presented. Crystal and magnetic structures and characteristic sizes have been determined by neutron diffraction for the antiferromagnetic core $(\mathrm{MnO})$ and the ferrimagnetic shell $\left(\gamma-\mathrm{Mn}_{2} \mathrm{O}_{3}\right)$. Remarkably, while the $\mathrm{MnO}$ core is found to have a $T_{N}$ not far from its bulk value, the magnetic order of the $\gamma-\mathrm{Mn}_{2} \mathrm{O}_{3}$ shell is stable far above $T_{C}$, exhibiting two characteristic temperatures, at $T \sim 40 \mathrm{~K}\left[T_{C}\left(\gamma-\mathrm{Mn}_{2} \mathrm{O}_{3}\right)\right]$ and at $T \sim 120 \mathrm{~K}\left[\sim T_{N}(\mathrm{MnO})\right]$. Magnetization measurements are consistent with these results. The stabilization of the shell moment up to $T_{N}$ of the core can be tentatively attributed to core-shell exchange interactions, hinting at a possible magnetic proximity effect.

DOI: 10.1103/PhysRevLett.102.247201

Magnetic nanoparticles are currently being extensively studied [1]. Among them, bimagnetic core-shell systems, where both the core and the shell are magnetic, are gaining increased interest due to appealing novel properties and promising applications, such as enhanced superparamagnetic blocking temperatures or tunable coercivities [2-10]. Most of the conventional bimagnetic core-shell nanoparticles have been obtained from the oxidation of transition metal nanoparticles, leading to a ferromagnetic (FM) core and the corresponding antiferromagnetic (AFM) or ferrimagnetic (FIM) shell [2,3]. In this case, the exchange coupling between the core and the shell gives rise to diverse effects such as loop shifts ("exchange bias") and coercivity enhancement $[2,3]$.

Recently so-called "inverted" core-shell systems with an AFM core and a FIM shell, e.g., $\mathrm{MnO} / \mathrm{Mn}_{3} \mathrm{O}_{4}$, have come into focus [11-14]. In this particular system the Curie temperature $\left(T_{C}\right)$ is below the Néel temperature $\left(T_{N}\right)$, in contrast to most of the exchange biased thin film and core-shell systems [2]. This doubly inverted (i.e., AFM core, not FM, and $T_{N}>T_{C}$ ) structure gives rise to a number of interesting effects, such as a nonmonotonic dependence of the loop shift on the core diameter and the existence of loop shifts above $T_{C}$ [11-13]. Importantly, the latter effect is different from the known enhancement of the blocking temperature in usual core-shell systems, which arises from the increase of the effective anisotropy of the FM core induced by the coupling to the AFM shell [15].
PACS numbers: 75.50.Tt, 61.05.F-, 75.30.Kz, 75.75.+a

In thin film systems consisting of two different magnetic materials the enhancement of $T_{N}$ or $T_{C}$ of one of the layers due to exchange interactions (i.e., a magnetic proximity effect) has been reported [16-22]. However, this effect has never been reported in nanoparticles.

In this Letter we present the study of inverted core-shell nanoparticles consisting of a $\sim 5 \mathrm{~nm}$ (sample $S$ ) or $\sim 17 \mathrm{~nm}$ (sample $L$ ) antiferromagnetic $\mathrm{MnO}$ core and a $\sim 5 \mathrm{~nm}$-thick ferrimagnetic $\gamma-\mathrm{Mn}_{2} \mathrm{O}_{3}$ shell [23]. It is found that the ordered magnetic moment of the $\gamma-\mathrm{Mn}_{2} \mathrm{O}_{3}$ shell remains finite far above $T_{C}$ of the $\gamma-\mathrm{Mn}_{2} \mathrm{O}_{3}$ shell and $T_{N}$ of the $\mathrm{MnO}$ core.

Neutron diffraction measurements were carried out at the diffractometer D20 of the Institute Laue-Langevin with a neutron wavelength of $1.305 \AA$ [24]. All diffraction patterns were analyzed using the FULLPROF program [25] based on the known crystal structures of the core and the shell. This method, in contrast to the so-called "matching mode," provides more stable refinement in the present case due to the strong overlapping of the principal peaks and the presence of small parasitic reflections. Magnetization measurements were carried out using a SQUID magnetometer.

The $\mathrm{MnO}$ core exhibits the expected $\mathrm{NaCl}$ structure. However, refinement of the occupancy factors shows that sample $S$ has defects in the Mn sites, with an occupancy factor of only 0.74(2). Sample $L$ exhibits the stoichiometric structure. Since the detected defects are only observed in the samples with smaller cores (i.e., with a large surface to 
volume ratio) it can be inferred that they should be at the $\mathrm{MnO}$ surface. Interestingly, the detection of defects is consistent with the model of $\mathrm{Mn}_{3} \mathrm{O}_{4}$ growing on $\mathrm{MnO}$ proposed by Berkowitz et al. [12]. These defects could lead to so-called uncompensated spins [26], which, in turn, could explain the large loop shifts observed in this type of nanoparticles [11-13].

The shell of the smaller particles has a spinel-type tetragonal structure with two types of voids: tetrahedral (eightfold $A$ position) and octahedral (16-fold $B$ position). From the profile analysis it follows that the shell has the structure formulas $\left(\mathrm{Mn}_{2 / 3}^{3+} \square_{1 / 3}\right)\left[\mathrm{Mn}^{3+}\right]_{2} \mathrm{O}_{4}$, which, in fact, corresponds to the defect structure, known as $\gamma-\mathrm{Mn}_{2} \mathrm{O}_{3}$ $[27,28]$, not the expected hausmannite $\left(\mathrm{Mn}^{2+}\right)\left[\mathrm{Mn}^{3+}\right]_{2} \mathrm{O}_{4}$ (or $\mathrm{Mn}_{3} \mathrm{O}_{4}$ ). Here, the parentheses and square brackets refer to the tetrahedral and octahedral voids, respectively, and the symbol $\square$ corresponds to vacancies. Note that for the larger particles the structure of the shell cannot be univocally established due to the relatively small diffraction signal.

The temperature dependence of the lattice parameter of the $\mathrm{MnO}$ cores is consistent with a well structured core [23,29-33]. Similarly, the lattice parameters of $\gamma-\mathrm{Mn}_{2} \mathrm{O}_{3}$ are also close to bulk values [23,27,28].

The diffraction lines are broadened with respect to the instrumental resolution, indicating that the correlation length is finite. However, from the neutron diffraction patterns the expected stresses appeared to be negligible within the experimental error. Thus, from the peak broadening the characteristic sizes of the nanoparticles were evaluated. The volume average diameter of the core and the characteristic size of the ferrimagnetic shell are 4.9(3)/4.7(3) and 17.0(5)/5(1) nm, at $170 \mathrm{~K}$, for samples $S$ and $L$, respectively. Note that the values in the brackets correspond to the error defined as the estimated standard deviation.

Using the known AFM order in bulk $\mathrm{MnO}$ and taking into account the trigonal distortion due to magnetostriction [34], the ordered magnetic moments in the $\mathrm{MnO}$ core were refined. The values of the saturated magnetic moment of $\mathrm{MnO}$ are smaller than the expected $5 \mu_{B}$ for the free $\mathrm{Mn}^{2+}$ ion [Fig. 1(a)], which can be explained partly due to the lower occupancy of the $\mathrm{Mn}^{2+}$ sites, that increases the effective valence state and consequently decreases the averaged magnetic moment, and partly by surface spin disordering [30]. The temperature dependence of the magnetic moment shows a continuous transition, in contrast with the first order transition in bulk, due to size effects [30]. Interestingly, $T_{N} \sim 130(9) \mathrm{K}$ (for sample $S$ ) and 120.9(2) (for sample $L$ ) are enhanced with respect to the bulk value of $117 \mathrm{~K}$ [29], similar to what was observed in $\mathrm{MnO}$ nanoparticles with different shapes and sizes confined within diverse porous media $[30,32]$.

$\gamma-\mathrm{Mn}_{2} \mathrm{O}_{3}$ nanoparticles are known to be ferrimagnetic with a $T_{C}$ of about $40 \mathrm{~K}$ with large coercivities at low

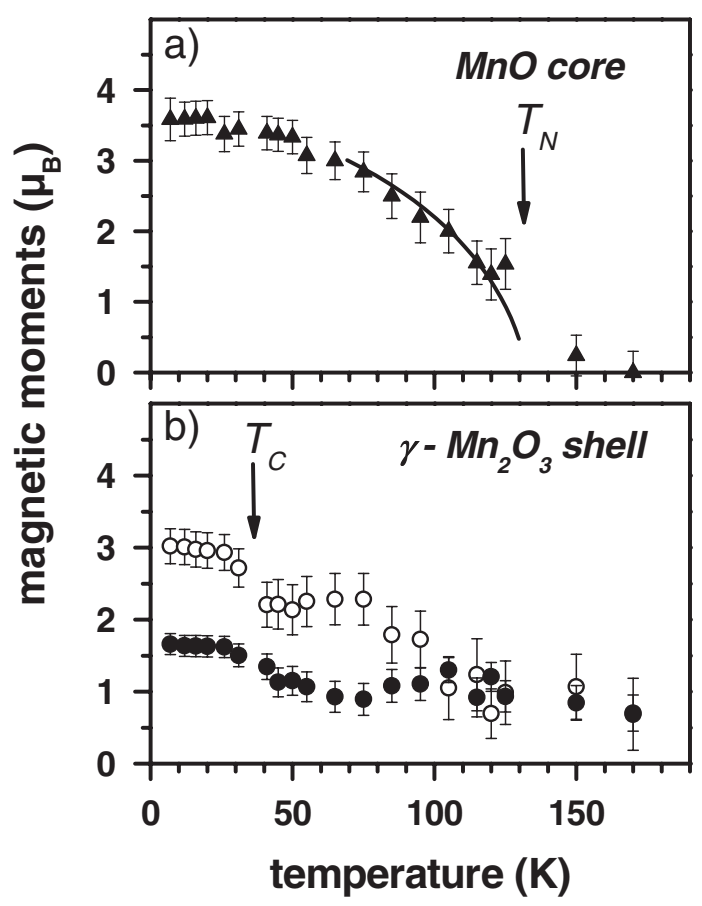

FIG. 1. Temperature dependence of the magnetic moment of (a) $\mathrm{Mn}^{2+}$ in $\mathrm{MnO}$ (the solid lines correspond to a power law fit) and (b) at the tetrahedral sites $(\bigcirc)$ and at the octahedral sites $(\bigcirc)$ of the $\gamma-\mathrm{Mn}_{2} \mathrm{O}_{3}$ for sample $S$. Arrows mark the magnetic transition temperatures.

temperatures [35]. However, the magnetic structure of $\gamma-\mathrm{Mn}_{2} \mathrm{O}_{3}$ is unknown. For the related hausmannite $\mathrm{Mn}_{3} \mathrm{O}_{4}$ structure, a complex magnetic order with a doubled nuclear cell has been reported [36]. However, we did not observe any superstructure magnetic reflections at low temperatures, implying that the magnetic cell coincides with the chemical one. Therefore we worked in the frame of the two-sublattice collinear ferrimagnetic model of Néel [37], similar to $\gamma-\mathrm{Fe}_{2} \mathrm{O}_{3}$. Any small distortions of such structure were beyond our statistic accuracy. The temperature dependence of the magnetic moment in the tetrahedral $(A)$ and the octahedral $[B]$ sites for sample $S$ (Fig. 1) exhibits two remarkable features. First, there is a large difference between the magnetic moments in $A$ and $B$ sites, and second, the moments are noticeably reduced with respect to the $4 \mu_{B}$ expected for the free $\mathrm{Mn}^{3+}$ ion. Although the orbital moment of the $\mathrm{Mn}^{3+}$ ion and the crystalline field (which is different for the $A$ and $B$ voids) could affect the value of the magnetic moment, similar effects have been observed in $\gamma-\mathrm{Fe}_{2} \mathrm{O}_{3}$, where $\mathrm{Fe}^{3+}$ does not possess orbital moment. In Néel-type order the effective exchange magnetic field in position $B$ from the surrounding magnetic moments is about twice weaker than those in position $A$. The spins which are more weakly coupled should be more disordered due to the breaking of local symmetry, and, consequently, their mean value should be smaller [38]. Hence, we attribute the observed 
difference of magnetic moments in the $\gamma-\mathrm{Mn}_{2} \mathrm{O}_{3}$ shell to this mechanism. Moreover, spin canting in the $\gamma-\mathrm{Mn}_{2} \mathrm{O}_{3}$ structure (similar to what is observed in $\gamma-\mathrm{Fe}_{2} \mathrm{O}_{3}$ shells [39]) could also contribute to the reduced moment, although due to high anisotropy of $\gamma-\mathrm{Mn}_{2} \mathrm{O}_{3}$ the canting is expected to be smaller than in $\gamma-\mathrm{Fe}_{2} \mathrm{O}_{3}$ and probably confined in the interface layers. Taking into account the number of tetrahedral and octahedral sites the net moment at $T=10 \mathrm{~K}$ per formula unit is about $\mu \sim 1.0(2) \mu_{B}$. Note that due to peak overlapping meaningful magnetic information for the shell can only be obtained for sample $S$.

The temperature dependence of the magnetic moment of both sublattices of the $\gamma-\mathrm{Mn}_{2} \mathrm{O}_{3}$ shell shows a kink around the known $T_{C}$ of this material $(40 \mathrm{~K}$ ) [arrow in Fig. 1(b)] and exhibits a finite moment even above $T_{C}$ (see also [23]). Since the error bars obtained from the refined profile are somewhat large, in Fig. 2 the intensity of the isolated magnetic peak for sample $S$, which is proportional to the square of magnetic moment, with the dominant contribution of the $\gamma-\mathrm{Mn}_{2} \mathrm{O}_{3}$ moments and a smaller contribution of the magnetic moments of the $\mathrm{MnO}$ core are shown. The last contribution, calculated from the profile analysis, is displayed in Fig. 2 separately. Indeed, the magnetic reflections from the shell have a sizable intensity even above $40 \mathrm{~K}$ in concordance with the magnetic moments obtained from the profile analysis. Note that the presence of magnetic signal above $T_{C}$ is consistent with recent magnetic results of Berkowitz et al. [13] and the presence of two maxima (around $T_{C}$ and $T_{N}$ ) in the $\mathrm{Mn}_{3} \mathrm{O}_{4}$ electron spin resonance linewidth observed in $\mathrm{MnO}-\mathrm{Mn}_{3} \mathrm{O}_{4}$ systems

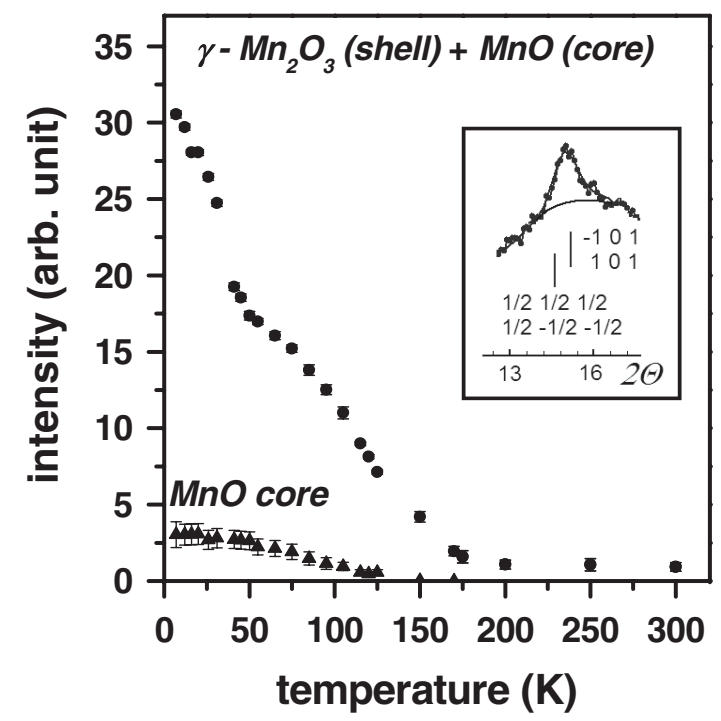

FIG. 2. Temperature dependence of the intensity of the magnetic peak at $15.4^{\circ}(\mathbf{O})$ for sample $S$. There are contributions from the $\mathrm{MnO}$ core: reflections $(1 / 2,1 / 2,1 / 2)$, $(1 / 2,-1 / 2,-1 / 2)$, and from the $\gamma-\mathrm{Mn}_{2} \mathrm{O}_{3}$ shell: reflections $(1,0,1)$ and $(-1,0,1)$. The contribution from the $\mathrm{MnO}$ calculated from the profile analysis is shown by $\boldsymbol{\Delta}$. Shown in the inset is the observed magnetic peak at $T=250 \mathrm{~K}$.
$[14,40]$. Remarkably, a second kink in the temperature dependence of the magnetic moment is observed around $T_{N}$ of $\mathrm{MnO}(\sim 130 \mathrm{~K})$ as highlighted by an arrow in Fig. 1(a). Surprisingly, the induced moment does not vanish at $T_{N}(\mathrm{MnO})$ and persists far above. Certainly, a weak peak at the position of the $\gamma-\mathrm{Mn}_{2} \mathrm{O}_{3}(1,0,1)$ reflection is clearly seen up to $300 \mathrm{~K}$ (inset in Fig. 2). A comprehensive $\mathrm{x}$-ray inspection at these angles does not show any coherent reflections. The peak broadening corresponds to the size of the nanoparticles composing the shell. Hence, we attribute this peak to the coherent magnetic scattering from the shell. The profile analysis renders a magnetic moment of about 1.3(3) $\mu_{B}$. Within the experimental accuracy, neither intensity nor line shape appear to vary with temperature. Nevertheless, a structural contribution to the peak at ca. $15^{\circ}$, due to a possible ordering of vacancies, cannot be completely ruled out, although x-ray diffraction does not show any clear coherent reflections.

To search for further evidence of the presence of potential proximity effects magnetization measurements were also carried out. However, due to the small core size of sample $S$ and the long characteristic measuring time of SQUID $\left(\tau_{m} \sim 100 \mathrm{~s}\right)$ no proximity effects are observable using magnetometry (see Fig. 3 and [23]). Interestingly, for sample $L$, both the temperature dependence of $M_{\mathrm{FC}}(T)-$ $M_{\mathrm{ZFC}}(T)$ (field cooled-zero field cooled) magnetization curves at $5 \mathrm{~T}$ (Fig. 3) and the saturation magnetization $M_{S}(T)$ [23] exhibit a considerable signal well above $T_{C}$ and up to about $T_{N}$ (in agreement with Berkowitz et al. [12]), which is consistent with a proximity effect. It should be taken into account that magnetization measurements are not equally as distinctive as neutron diffraction measurements (where the signal comes solely from the $\gamma-\mathrm{Mn}_{2} \mathrm{O}_{3}$ shell), since in this case uncompensated spins in the $\mathrm{MnO}$ could also contribute to the magnetic signal.

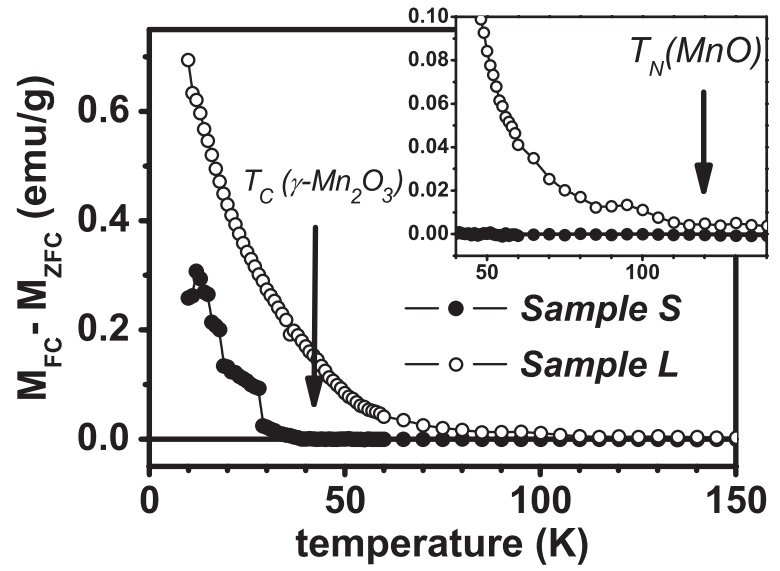

FIG. 3. Temperature dependence of the difference between the FC and ZFC magnetizations, $M_{\mathrm{FC}}-M_{\mathrm{ZFC}}$, for samples $S(\mathbf{O})$ and $L(\bigcirc)$. Shown in the inset is an enlargement of the high temperature region. The error bars are smaller than the size of the symbol, and the solid lines are guides for the eye. 
Interestingly, in contrast to thin film AFM/FM systems, where magnetic proximity effects are observed in the AFM (since $T_{C}>T_{N}$ ), in the $\mathrm{MnO} / \gamma-\mathrm{Mn}_{2} \mathrm{O}_{3}$ system induced moments are observed in the FM shell. We attribute the observed stable magnetic moment in the $\gamma-\mathrm{Mn}_{2} \mathrm{O}_{3}$ shell well above $T_{C}$ to the interface exchange coupling between the $\mathrm{MnO}$ core and the shell, as has been experimentally and theoretically observed in film systems [16-22,41,42], that can tentatively be considered as a magnetic proximity effect. Namely, although uncompensated spins may play a role in the proximity effect [12], the exchange field of each sublattice of the AFM core, which penetrates a few atomic layers, stabilizes the magnetic structure of the sublattices of the FIM shell [41,42]. These results are in agreement with the theoretical results in bilayer thin films from Jensen et al. who predicted an increase of the temperature range of stable moments to above $T_{C}$ in AFM/FM systems with $T_{N}>T_{C}$ [41]. Probably both the moderately strong core-shell interface exchange coupling (evidenced by the large exchange bias observed in these systems [1113]) and the small shell thickness contribute to make the effect more evident. The possible persistence of a magnetic moment in $\gamma-\mathrm{Mn}_{2} \mathrm{O}_{3}$ above $T_{C \text {, shell }}$ and $T_{N \text {,core }}$ is not clear at present, although it could be related to oxygen vacancies in $\gamma-\mathrm{Mn}_{2} \mathrm{O}_{3}$, as has been proposed [43] for other transition metal oxides exhibiting persisting magnetic signal well above their corresponding transition temperatures [43-45].

In conclusion, the magnetic order and temperature dependence of the magnetic moments in the doubly inverted "core-shell" $\mathrm{MnO} / \gamma-\mathrm{Mn}_{2} \mathrm{O}_{3}$ system were determined. It is found that an induced moment in the $\gamma-\mathrm{Mn}_{2} \mathrm{O}_{3}$ shell persists above its $T_{C}$ due to the exchange coupling with the $\mathrm{MnO}$ core (i.e., a likely magnetic proximity effect).

The work was supported by the Russian Foundation for Basic Researches (Grant No. N07-02-00608), the Spanish MICINN (Grants No. MAT2007-66302-C02 and No. CSD2006-00012 Consolider-Ingenio 2010), and the Institut Català de Nanotecnologia. J. N. thanks M. Kiwi and J. Borchers for fruitful discussions. We acknowledge the ILL for the provision of neutron beam time.

[1] A. H. Lu, E. L. Salabas, and F. Schuth, Angew. Chem., Int. Ed. 46, 1222 (2007).

[2] J. Nogués et al., Phys. Rep. 422, 65 (2005).

[3] O. Iglesias, A. Labarta, and X. Batlle, J. Nanosci. Nanotechnol. 8, 2761 (2008).

[4] M. Casavola et al., Eur. J. Inorg. Chem. 6 (2008) 837.

[5] W. Liu, W. Zhong, and Y. W. Du, J. Nanosci. Nanotechnol. 8, 2781 (2008).

[6] E. Eftaxias, M. Vasilakaki, and K. N. Trohidou, Mod. Phys. Lett. B 21, 1169 (2007).

[7] G. Salazar-Alvarez et al., J. Mater. Chem. 17, 322 (2007).

[8] J. Nogués et al., Phys. Rev. Lett. 97, 157203 (2006).
[9] X. S. Liu et al., Appl. Phys. A 77, 673 (2003).

[10] H. Zeng et al., Appl. Phys. Lett. 85, 792 (2004).

[11] G. Salazar-Alvarez et al., J. Am. Chem. Soc. 129, 9102 (2007).

[12] A. E. Berkowitz et al., Phys. Rev. B 77, 024403 (2008).

[13] A. E. Berkowitz et al., J. Phys. D 41, 134007 (2008).

[14] I. Djerdj et al., J. Phys. Chem. C 111, 3614 (2007).

[15] D. Givord, V. Skumryev, and J. Nogués, J. Magn. Magn. Mater. 294, 111 (2005).

[16] C. A. Ramos et al., Phys. Rev. Lett. 65, 2913 (1990).

[17] P. J. van der Zaag et al., Phys. Rev. Lett. 84, 6102 (2000).

[18] K. Lenz, S. Zander, and W. Kuch, Phys. Rev. Lett. 98, 237201 (2007).

[19] V. V. Volobuev, A. N. Stetsenko, and J. van Lierop, J. Appl. Phys. 103, 07C905 (2008).

[20] E. N. Abarra et al., Phys. Rev. Lett. 77, 3451 (1996).

[21] J. A. Borchers et al., Phys. Rev. Lett. 70, 1878 (1993).

[22] J. van Lierop et al., Phys. Rev. B 75, 134409 (2007).

[23] See EPAPS Document No. E-PRLTAO-103-004927 for the details of the synthesis of the nanoparticles, supplementary figures that show the temperature dependence of the $\mathrm{MnO}$ and $\gamma-\mathrm{Mn}_{2} \mathrm{O}_{3}$ lattice parameters, the total moment of $\gamma-\mathrm{Mn}_{2} \mathrm{O}_{3}$ for sample $S$, the magnetic measurements and the temperature dependence of $M_{S}$ for samples $L$ and $S$. For more information on EPAPS, see http:// www.aip.org/pubservs/epaps.html.

[24] T. C. Hansen et al., Meas. Sci. Technol. 19, 034001 (2008).

[25] J. Rodriguez-Carvajal, Physica (Amsterdam) 192B, 55 (1993); http://www.ill.eu/sites/fullprof.

[26] K. Takano et al., Phys. Rev. Lett. 79, 1130 (1997).

[27] J. B. Goodenough and A. L. Loeb, Phys. Rev. 98, 391 (1955).

[28] D. Bonnenberg and H. P. J. Wijn, in Magnetic and Other Properties of Oxides and Related Compounds, edited by K.-H. Hellwege and A. M. Hellwege, Landolt-Bornstein, Group III, Vol. 4b, Pt. B (Springer-Verlag, Berlin, 1970).

[29] B. Morosin, Phys. Rev. B 1, 236 (1970).

[30] I. V. Golosovsky et al., Phys. Rev. Lett. 86, 5783 (2001).

[31] I. V. Golosovsky et al., Phys. Rev. B 74, 054433 (2006).

[32] I. V. Golosovsky et al., Phys. Rev. B 72, 144409 (2005).

[33] I. V. Golosovsky et al., Phys. Rev. B 74, 155440 (2006).

[34] W. L. Roth, Phys. Rev. 110, 1333 (1958).

[35] S. H. Kim et al., J. Korean Phys. Soc. 46, 941 (2005).

[36] G. B. Jensen and O. V. Nielsen, J. Phys. C 7, 409 (1974).

[37] L. Néel, Ann. Phys. (Paris) 3, 137 (1948).

[38] I. V. Golosovsky et al., JETP Lett. 83, 298 (2006).

[39] L. Theil Kuhn et al., J. Phys. Condens. Matter 14, 13551 (2002).

[40] M. S. Seehra and G. Srinivasan, J. Appl. Phys. 53, 8345 (1982).

[41] P. J. Jensen, H. Dreysseé, and M. Kiwi, Eur. Phys. J. B 46, 541 (2005).

[42] A. S. Carriço and R. E. Camley, Phys. Rev. B 45, 13117 (1992).

[43] D. P. Dutta et al., Nanotechnology 19, 245609 (2008).

[44] C. Nethravathi et al., J. Phys. Chem. B 109, 11468 (2005).

[45] A. Sundaresan and C. N. R. Rao, Nano Today 4, 96 (2009). 